
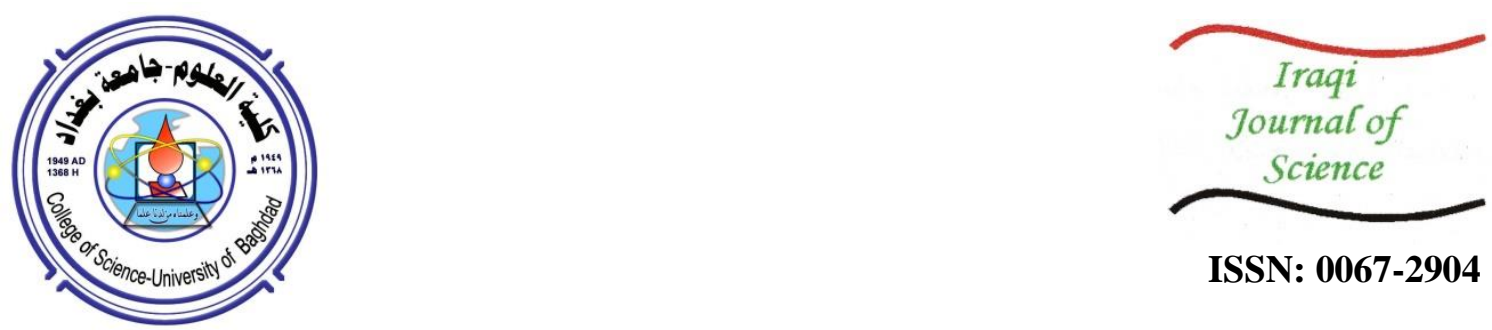

ISSN: 0067-2904

\title{
Experimental Investigation for Some Properties of PMMA Denture Base Strengthened by Different Nanoadditives
}

\author{
Zaynab N. Rasheed Alraziqi, Hadeel Salah Mansoor \\ Applied Science Department, University of Technology, Baghdad, Iraq \\ Received: 12/11/2019 \\ Accepted: 29/4/2020
}

\begin{abstract}
In dental and medical applications, poly-methyl methacrylate (PMMA) has been widely accepted due to the excellent biocompatibility and easy fabrication. Yet, some of the physical and mechanical characteristics of this compound are considered inferior. Seven groups of PMMA nano-composite samples were reported to be fabricated at laboratory temperature $\left(\mathbf{2 7}^{\circ}\right)$. These samples could be used in manufacturing the complete or partial maxillary denture base. The aim of this research is to prepare nanocomposite materials which consist of PMMA as a matrix material and two different types of powder (prepared nanoparticles of $\mathrm{SnO}_{2}$ and natural egg shell powder (ESP)) as strengthening materials. The selected additives were used in many cases as pure or hybrid composites, specifically with weight percentage ratios of 1 and $2 \mathrm{wt} \%$. Several analytical tests, namely AFM, FTIR, XRD and SEM, were used on the prepared nanoparticles. In addition, several tests were applied to assess the mechanical behavior before and after the reinforcement, including thermal conductivity, Vickers micro-Hardness, and water absorption. The results showed that the maximum amount of energy absorption in the composites was $1 \%$ at different types of additives, while a significant increase in thermal conductivity was recorded as the $\mathrm{SnO}_{2} \mathrm{NPs}$ percentage was increased. For surface micro-hardness, an obvious trend of increase was observed with the increase in additive percentage. The highest measured values of micro-hardness (19.59VHS and 13.30VHS) were recorded for the pure composites of $2 \% \mathrm{ESP}$ and $2 \% \mathrm{SnO}_{2}$, respectively. The results of water absorption test showed that higher percentages of ESP and $\mathrm{SnO}_{2}$, separately, within the pure composite resulted in higher water absorption capacity and an increased value of diffusion coefficient (D).
\end{abstract}

Keywords: PMMA, dental nano-composite, $\mathrm{SnO}_{2} \mathrm{NPs}$, egg shell powder (ESP), thermal conductivity, Vickers-hardness.

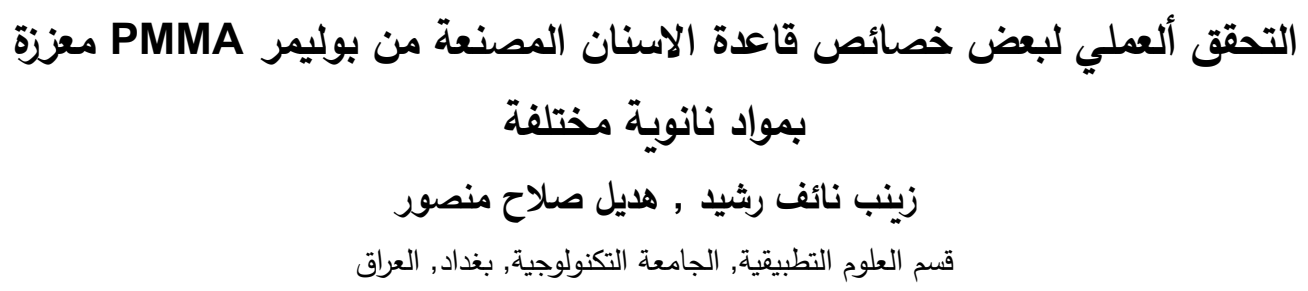

*Email: 100123@uotechnology.edu.iq 


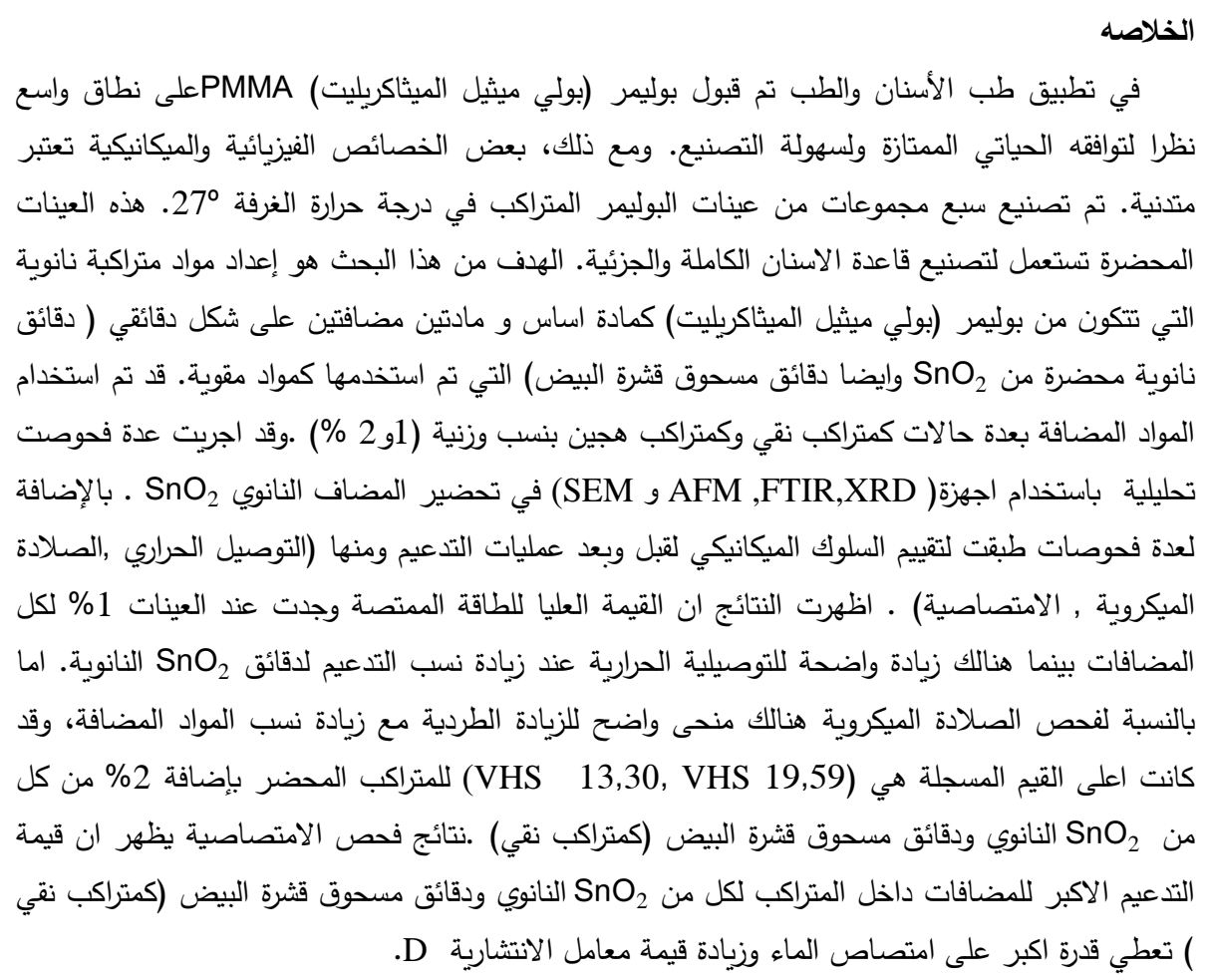

\section{Introduction}

Since 1937, PMMA has been used as a denture base material. Many features made this polymer desirable to be used in the fabrication of removable complete and partial dentures. Reasonable cost, esthetic properties, ease of manipulation, light weight, and excellent biocompatible material are the most supporting features to render this polymer the dominantly applied in this field $[1,2]$

There were few, but important, disadvantages involved in the utilization of PMMA, such as poor strength particularly under fatigue failure during mastication. Also, low thermal conductivity of PMMA, approximately $0.2 \mathrm{w} / \mathrm{m} /{ }^{\circ} \mathrm{c}$ [3], poses several problems in edentulous patients who wear full dentures. For example, in these cases, the palate is partially covered by the denture base; consequently, the thermal characteristics of the denture material affect the ability of the user to sense transient temperature changes at the palate. Therefore, these disadvantages in the physical characteristics of the material may affect the patient's acceptance of denture prostheses by altering sensory experience of food during mastication [4]. In addition, thermal characteristics are considered as essential factors affecting the gustatory response [5].

Due to of the importance of mechanical and physical properties of denture base material in dentistry, scientists modified PMMA by reinforcement through the addition of different particles and fillers. Nano-scale reinforcement agents provide new mechanical and physical properties, leading to the production of new polymeric nanocomposites as a promising new class of substances [6-8]. Many researchers investigated the effects of the addition of several nanoparticles on the mechanical and physical properties of PMMA. Nano aluminium oxide $\left(\mathrm{Al}_{2} \mathrm{O}_{3}\right)$ and nano hydroxyapatite (HA) has been used as reinforcement materials. Scientists investigated the influence of these additions on the mechanical characteristics of PMMA for dental applications. The results revealed better mechanical properties as compared to those of the pure PMMA [9]. In addition, $\mathrm{TiO}_{2}$ was used several times to enhance some properties of PMMA, including tensile and flexural strength [10,11]. The effects of the addition of silver, copper and aluminum powders to denture base material in different volume fractions were also investigated. Many studies were carried out to enhance the thermal properties of the acrylic denture base, for example through the addition of whiskers to PMMA matrix [12]. The addition of $\mathrm{Al}_{2} \mathrm{O}_{3}$ in different weight fractions was investigated to evaluate some mechanical and physical properties, such as thermal conductivity $[13,14,15]$. Moreover, natural powders at different sizes were investigated as additives to enhance some of the pure resin characteristics $[16,17]$.

In this study, synthesis of $\mathrm{SnO}_{2} \mathrm{NPs}$ using sol-gel method was accomplished to be used as a nanoadditive to denture material. The selection of these specific particles was due to their high thermal 
conductivity and good biocompatibility. Also, these NPs are in the form of white powder which is acceptable for esthetical purposes. Furthermore, ESPs were prepared and processed for the same purpose. The use of waste materials, such as ESPs, in polymer composite fabrication is progressively developing due to environmental and economic apprehensions. The present study evaluated the effects of nano- $\mathrm{SnO}_{2}$ and ESPs additives of PMMA, separately and combined (experimental case), as compared to those of the unreinforced PMMA (control case). Moreover, thermal conductivity and micro-hardness were tested for all prepared samples in different addition percentages for both cases (pure and hybrid).

\section{Materials and methods}

\subsection{PMMA preparation}

Self- cure, multifunctional, self-polymerizing acrylic PMMA (Spofa Dental, Czech Republic) was utilized in this research as a matrix material The standard volumetric mixing ratio for self-cure PMMA acrylic resin was 3:1 (3 parts of powder: 1 part of liquid) according to the manufacturer's instructions. Using hand lay-out technique. The mixture was stirred until the dough stage at room temperature. Then, the final dough was poured into a glass mould. For solidification purposes, this mixture was left at room temperature for about 3 hours. Post-cure process performed by placing the released sheet from the mould in an oven at $55{ }^{\circ} \mathrm{C}$ for additional 1 hour.

\subsection{Egg-shell powder preparation.}

Figure-(1a and b) demonstrates the collected egg-shells from local households before and after the preparation procedure. To obtain egg-shell powder, the shells were first washed with demineralised water to remove any other objects, confer moisture, and eliminate the thin outer membrane. The eggshells were then subjected to sun-drying for duration of 48 hours. The dried egg-shells were pulverized to obtain a fine powder, as illustrate in Figure-1b, using of a grain miller at $250 \mathrm{rpm}$. The sieved ESPs were heated until $100 \mathrm{C}^{\circ}$ for few hours to remove any moisture and transferred to a desiccator to avoid contact with atmosphere. Figure-2 illustrates the particle size and distribution of ESP as analyzed by using particle size analyzer, where the effective diameter was found to be $0.973 \mu \mathrm{m}$.
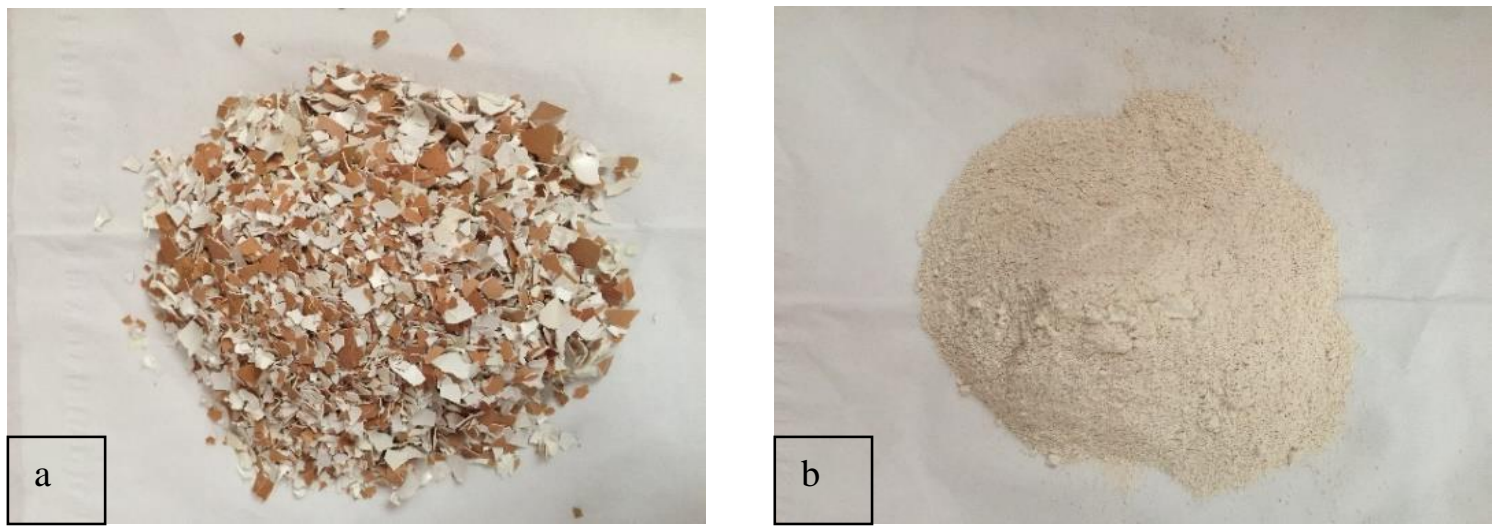

Figure 1(a)-collected egg-shell before grinding process (b) Egg-shell powder after grinding process.
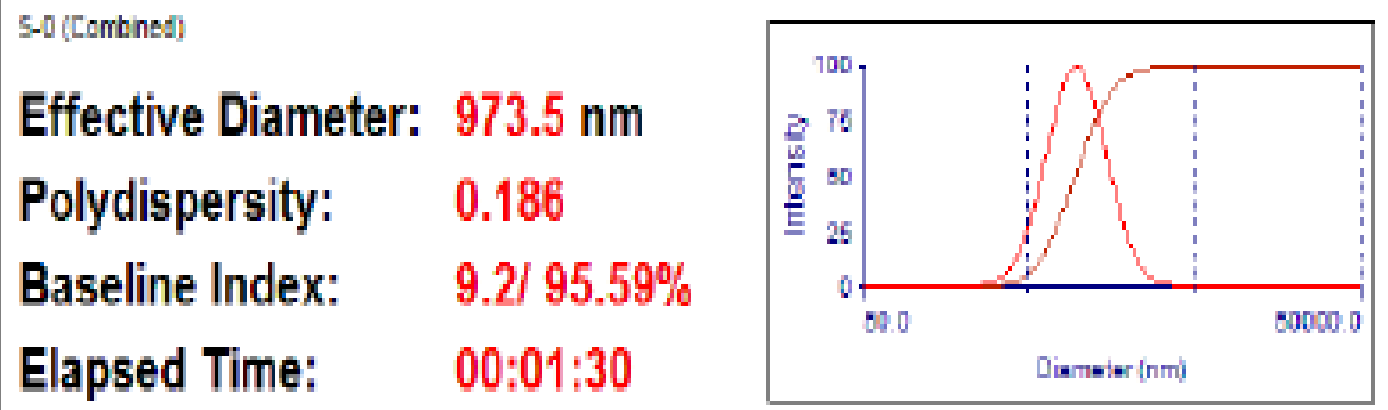

Lognomal Distribution

Figure 2-Egg shell particle size test (effective diameter $0.973 \mu \mathrm{m}$ ) 


\section{3 $\mathrm{SnO}_{2} \mathrm{NPs}$ preparation}

Tin dioxide $\left(\mathrm{SnO}_{2}\right)$ nanoparticles were prepared by the titration (sol-gel) method [18]. An amount of $2 \mathrm{~g}$ of stannous chloride dehydrate $\left(\mathrm{SnCl}_{2} .2 \mathrm{H}_{2} \mathrm{O}\right.$, Aldrich, purity $\left.99.8 \%\right)$ was first dissolved in water. The solution was stirred in a beaker with a magnetic stirrer for 20 min, until it became colorless. Then, ammonium hydroxide solution $\left(\mathrm{NH}_{4} \mathrm{OH}\right)$ was added slowly until the $\mathrm{pH}$ value of the solution approached $\approx 8$. Through centrifugation, the precipitate was washed with distilled water for 5 times, then with absolute ethanol for 2 times. Finally, the precipitate was dried at $200{ }^{\circ} \mathrm{C}$ for $90 \mathrm{~min}$ to produce a pale yellow powder. The suggested reactions between stannous chloride dihydrate and ammonium hydroxide solution to produce $\mathrm{SnO}_{2}$ are as follows [19, 20]:

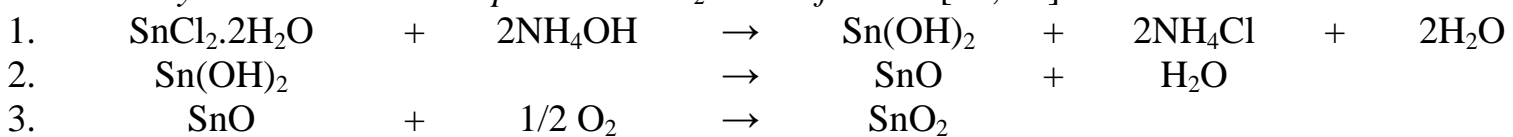

The final reaction is as follows:

$\mathrm{SnCl}_{2} \cdot 2 \mathrm{H}_{2} \mathrm{O}+2 \mathrm{NH}_{4} \mathrm{OH}+1 / 2 \mathrm{O}_{2} \rightarrow \mathrm{SnO}_{2}+2 \mathrm{NH}_{4} \mathrm{Cl}+3 \mathrm{H}_{2} \mathrm{O}$

Atomic force microscope (AFM, scanning probe microscopy (CSPM -5000) instrument)) was then used to measure the average diameter of the nanoparticles and their distribution. . Figure- 3 shows the size and distribution for the prepared nanoparticles. A three dimensional image and surface morphology of $\mathrm{SnO}_{2} \mathrm{Nps}$, annealed at $200{ }^{\circ} \mathrm{C}$, are demonstrated in Figure-3. The average grain size of these NPs was found to be $82 \mathrm{~nm}$.
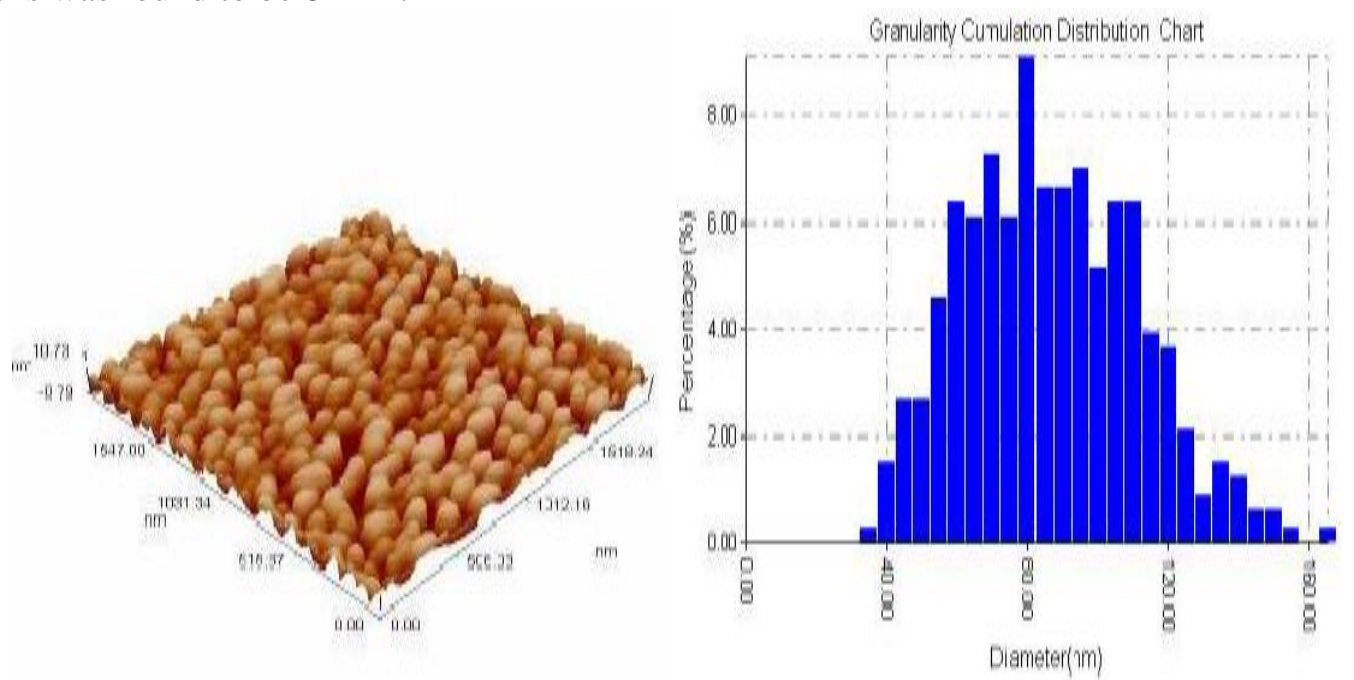

Figure 3-3D image of Atomic force microscopic and average diameter graph of $\mathrm{SnO}_{2} \mathrm{Nps}$ with annealing at $200^{\circ} \mathrm{C}$ for $90 \mathrm{~min}$.

\subsection{Preparation of ESPs/PMMA and $\mathrm{SnO}_{2} / \mathrm{PMMA}$ nano-composites.}

Nano-composite samples were prepared by mixing PMMA polymer and two types of nanoparticles. Both of the prepared ESPs powder and the synthetic $\mathrm{SnO}_{2}$ were used for the preparation of the testing samples. Table-1 lists the weight proportions of the materials utilized in these experiments. All samples were prepared using hand lay- up technique at room temperature. The dimensions of the used mould were $(10 * 7 * 0.5) \mathrm{mm}$. After pouring the (PMMA liquid-additive) mixture from the baker to the prepared mould, it was necessary to cover the mould by a metallic plate with a similar size, in order to obtain a smooth sample surface and to prevent the entry of gases vapour into PMMA sample during the curing. The nano-additive was mixed with the specific amount of PMMA powder for each sample, according to ratios described in Table-1. The PMMA-additive mixture solution was then poured slowly into the preparation baker with continuous mixing. Lastly, the prepared mixture was poured into the same mould used above and left to dry, following the same procedure which was applied for pure PMMA fabrication. The prepared samples were then cut into the standard dimensions of the selected tests, according to ISO-22007 standard. 
Table 1-Composition of different nanoparticle additive-reinforced composites

\begin{tabular}{|c|c|c|c|c|c|c|}
\hline \multirow{2}{*}{$\begin{array}{c}\text { Sample } \\
\text { code }\end{array}$} & \multicolumn{2}{|c|}{ Material proportion $\%(\mathrm{w} / \mathrm{w})$} & \multicolumn{2}{|c|}{ Weight of } & \multicolumn{2}{|c|}{$(\mathrm{g})$} \\
\cline { 3 - 7 } & PMMA & $\mathrm{SnO}_{2}$ & $\begin{array}{c}\text { eggshell } \\
\text { powder }\end{array}$ & PMMA (g) & $\mathrm{SnO}_{2}$ & eggshell powder \\
\hline $\mathrm{A}$ & 100 & - & - & 41.58 & - & - \\
\hline $\mathrm{B}$ & 99 & 0.5 & 0.5 & 41.10 & 0.21 & 0.21 \\
\hline $\mathrm{C}$ & 99 & - & 1 & 41.10 & - & 0.42 \\
\hline $\mathrm{D}$ & 99 & 1 & - & 41.10 & 0.42 & - \\
\hline $\mathrm{E}$ & 98 & 1 & 1 & 40.74 & 0.41 & 0.41 \\
\hline $\mathrm{F}$ & 98 & - & 2 & 40.74 & - & 0.83 \\
\hline $\mathrm{G}$ & 98 & 2 & - & 40.74 & 0.83 & - \\
\hline
\end{tabular}

\section{Testing procedure}

\subsection{Characterization of $\mathrm{SnO}_{2}$ nanoparticles}

$\mathrm{X}$-ray diffraction (XRD) type (6000 Shimadzu) with an X-ray powder diffractometer $\left(\mathrm{CuK}_{\alpha}\right.$ radiation and a wavelength, $\lambda=1.54060$ ) were employed to identify the crystalline structure of the prepared $\mathrm{SnO}_{2}$ nanoparticles. Also, Fourier transforms infrared spectroscopy (FTIR) was used to analyze and identify the functional groups of the prepared nanoparticles, the setting of $\mathrm{KBr}$ disc used with 8400S Shimadzu in the range of 4000-400 $\mathrm{cm}^{-1}$. The morphology of the prepared $\mathrm{SnO}_{2}$ nanoparticles was studied utilizing a scanning electron microscope (SEM) (INSPECT S50; FEI, Hillsboro, OR, USA), with an accelerating voltage of $30 \mathrm{kV}$ and magnification of 10000x.

\subsection{Thermal conductivity test}

Thermal conductivity indicates the heat transported from high to low temperature regions of a substance. Thermal conductivity devices measure the ability of such material to transfer heat. It also can be defined by the following expression:

$$
J_{E}=-K \frac{d \tau}{d x}
$$

where $J_{E}$ represents the heat flux, or heat flow, per unit time per unit area (where the area is considered as that perpendicular to the flow direction). K represents the thermal conductivity property and $\frac{d \tau}{d x}$ is the temperature gradient through the conductive medium. Thermal property was tested in this work using Lee's disc device. The heat in which flows inside the cross-section area of the sample per unit time was measured using following equation [21]:

$K\left[\frac{T B-T A}{\mathrm{dS}}\right]=e\left[T_{A}+\frac{2}{r}\left(d_{A}+1 / 4 d_{S}\right) T_{A}+1 / 2 r d_{S} T_{B}\right]$

where $T_{(A, B, C)}$ are the temperatures of $A, B$ and $C$ brass discs, whereas $d_{(A, B, C)}$ are their thickness values, respectively. I represents current value through the electrical circuit, while $\mathrm{V}$ is the voltage supplied. Then, thermal conductivity $K(\mathrm{~W} / \mathrm{m} . \mathrm{K})$ was measured using the following equation:

\subsection{Vickers Hardness Test}

$$
I V=\pi r^{2} e\left(T_{A}+T_{B}\right)+2 \pi r e\left[d_{A} T_{A}+d_{s} 1 / 2\left(T_{A}+T_{B}\right) d_{s} T_{S}+d_{c} T_{c}\right]
$$

Generally, the hardness of a specific material surface is a measurement of the deformation resistance. All prepared samples were subjected to Vickers hardness, also called micro-hardness test, by which the surface hardness (HV) was determined. Vickers hardness testing machine of a digital type (HVS -1000, Laryree technology Co.Ltd, China) was utilized. The setting load was 150 grams for 15 seconds (ADA Specification No.27). The average magnitude of three readings was recorded for 
each specimen. Vickers micro-hardness indenter is made of diamond indenter which is pressed onto the surface of the prepared sample material to cause a square-based pyramid indentation, which represents the surface resistance.

\subsection{Water absorption test}

This test is considered as critical for polymeric materials that come in contact with water, due to their susceptible nature to water absorption. Water absorption leads to internal stresses, degradation, and dimensions instability, finally causing easy crack and fracture of denture base [22]. This test was performed according to ASTEM D 1037-99. All the prepared samples (control and experimental) were soaked in mineral water at ambient temperature for 1 hour and a periodic weight measurement was performed each $15 \mathrm{~min}$ using a digital balance of 0.0001 resolution. Before weighing, all samples were removed from water and wiped to remove any surface water. Finally, diffusion coefficient (D) for all the prepared samples was evaluated at the same circumstances (liquid, temperature). D could be obtained from the following equation:

$$
A=\pi\left[\frac{\mathrm{Sd}}{4 \mathrm{M}_{\infty}}\right]^{2}
$$

where $S$ represents the slope between the weight gain and square time root, $d$ is sample thickness, and $\mathrm{M}_{\infty}$ is the highest mass gain determined for the sample. The weight gain of the samples was measured as the increase in weight $(\%)$ using the equation below:

Increase in weight $\%=$ (wet sample weight - sample weight $) /$ sample weigh $\mathrm{x} 100$

\section{Results and discussion}

\subsection{Characterization of $\mathrm{SnO}_{2}$ nanoparticles using XRD, FTIR, and SEM tests}

The X-ray diffraction test results of $\mathrm{SnO}_{2}$ nanoparticles, annealed at $200^{\circ} \mathrm{C}$, are shown in Figure-4. The results show polycrystalline phases, when compared with the card (JCPDS card no. 41-1445) [23]. The samples display four diffraction peaks at $2 \Theta=26.71,33.94,37.83$, and $51.99^{\circ}$, which correspond to the diffraction planes (110), (101) and (211) respectively.

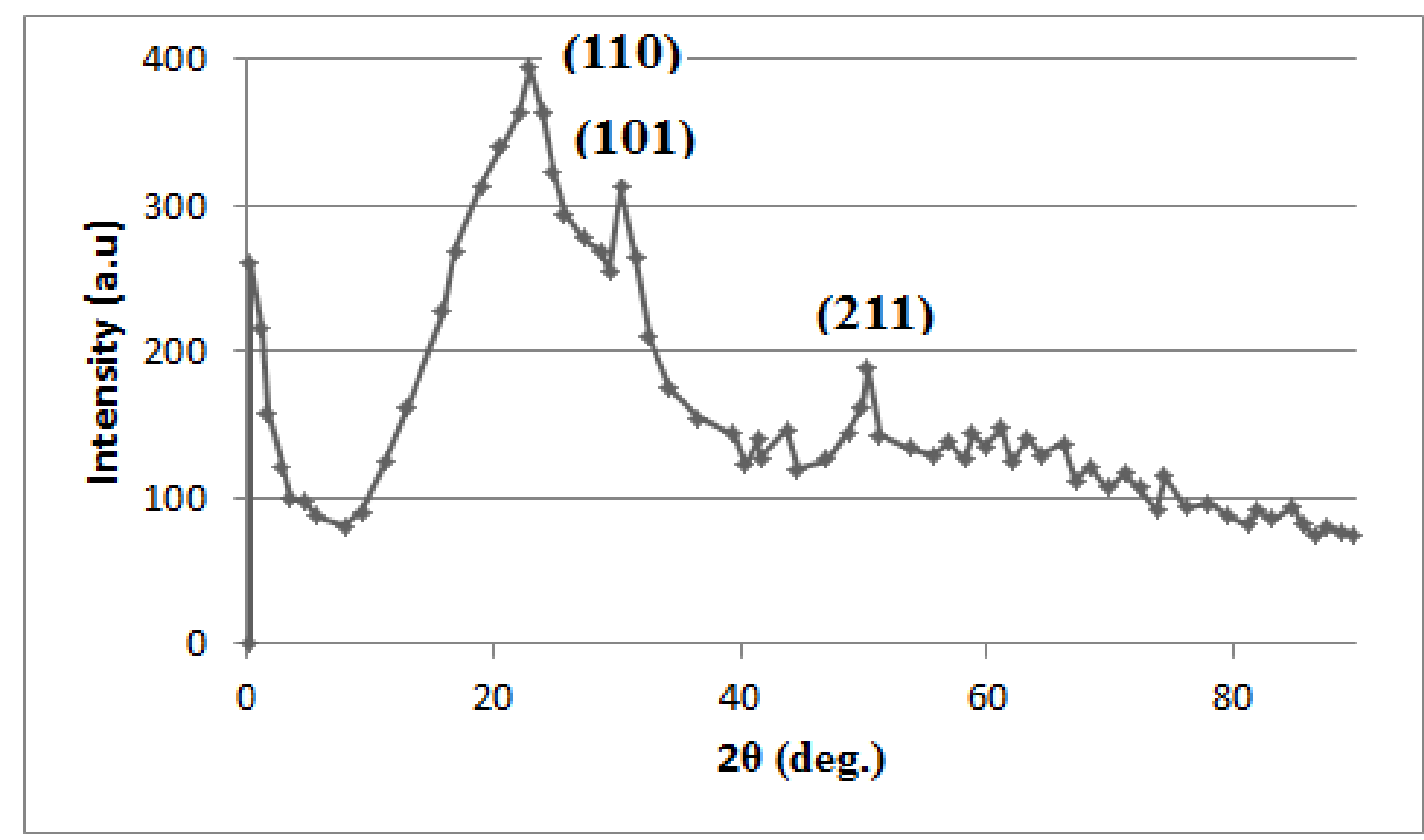

Figure 4-XRD pattern of $\mathrm{SnO}_{2} \mathrm{NPs}$ annealed at $200^{\circ} \mathrm{C}$.

Figure-5 illustrates The FTIR spectra of $\mathrm{SnO}_{2}$ NPs annealed at $200{ }^{\circ} \mathrm{C}$. The highest wave number region (peak at $3400 \mathrm{~cm}^{-1}$ ) of the spectrum is corresponding to stretching vibration of hydroxyl groups $(\mathrm{OH})$. The wave number region (peak at $1631 \mathrm{~cm}^{-1}$ ) is attributed to bending vibration of hydroxyl groups in water molecules. On the other hand, the lowest wave number region (peaks around $543 \mathrm{~cm}^{-}$ ${ }^{1}$ ) corresponds to $\mathrm{Sn}-\mathrm{O}-\mathrm{Sn}$ bonds [24] 


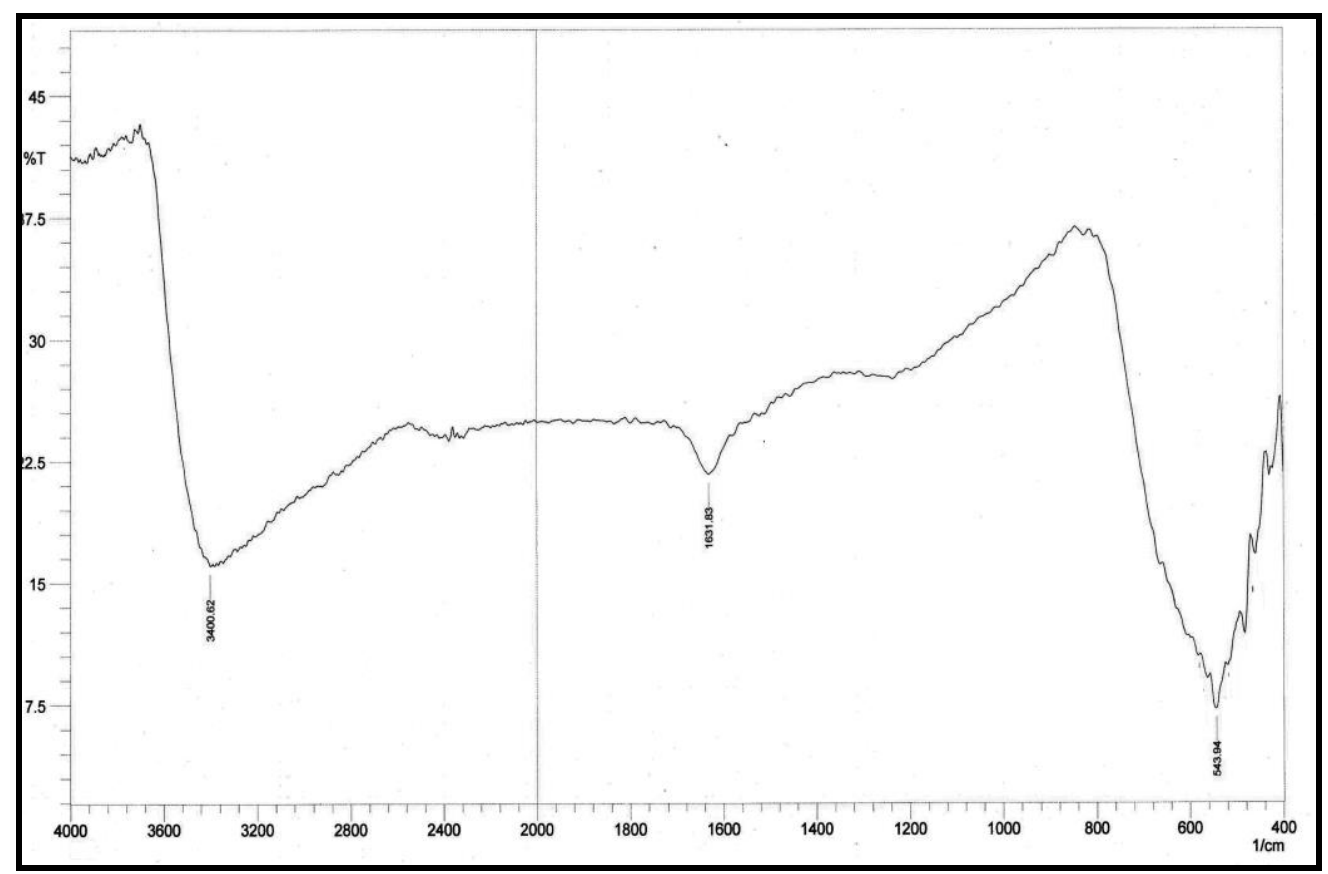

Figure 5-FTIR transmittance spectrum of $\mathrm{SnO}_{2} \mathrm{NPs}$ annealed at $200{ }^{\circ} \mathrm{C}$ for $90 \mathrm{~min}$.

The SEM images of the thin film of $\mathrm{SnO}_{2}$ NPs annealed at $200^{\circ} \mathrm{C}$ are illustrated in Figure-6. It is clear that the NPs agglomerate as clusters over the surface, which may be related to the effect of annealing temperature on.

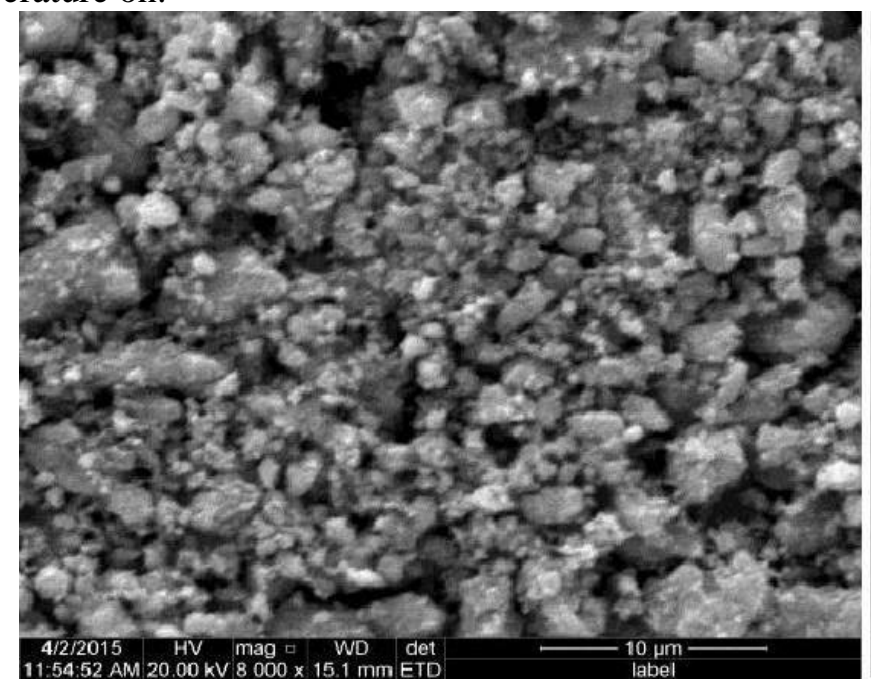

Figure 6-SEM images of $\mathrm{SnO}_{2}$ thin film nanoparticles annealed at 200 for $90 \mathrm{~min}$.

\subsection{Evaluation of thermal conductivity and absorbed energy}

In this research, six nano-composite samples reinforced by two kinds of nano-particles $\left(\mathrm{SnO}_{2}\right.$ and ESP) were produced and tested for both pure and hybrid reinforcement in a selected weight percentages. As mentioned before for all samples, the used matrix was PMMA and the additives used were prepared from natural and synthetic sources in various contents, as described in Table-1. Figure7 represents the results of absorbed energy magnitude for the different samples prepared. It is clear that B, C, and D samples had the highest amount of energy absorption and that could be related to the $1 \%$ weight percentage of the additive used. On the other hand, sample D showed the highest value of absorption among the samples due to the lowest nano-size of the used $\mathrm{SnO}_{2} \mathrm{NPs}$. The higher additive percentage resulted in a noticeable decrease in the measured value of absorption. 


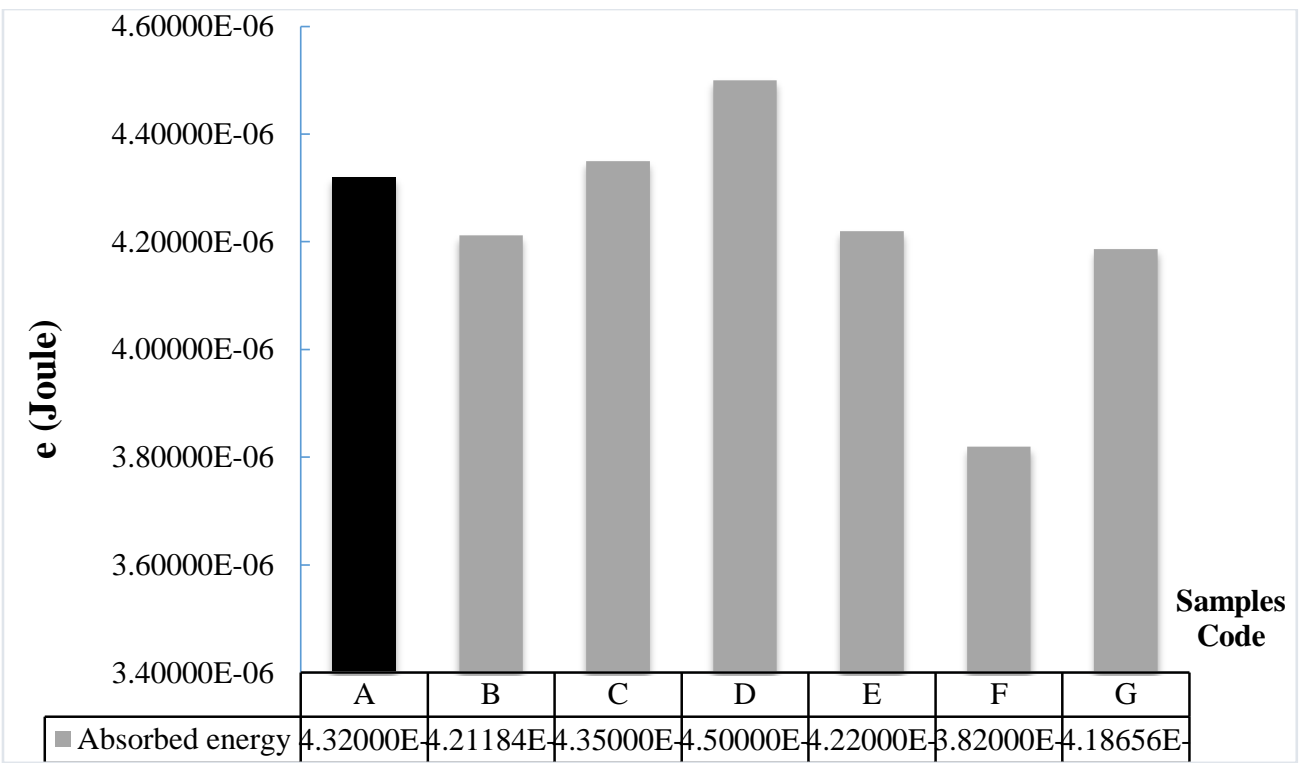

Figure 7-Comparison of the determined absorbed energy for the prepared composite with $\mathrm{SnO}_{2} \mathrm{NPs}$ and natural egg shell powder (ESP) at selected percentage as in Table-1.

Figure-8 presents thermal conductivity results for all samples. The observed results demonstrated that the addition of both types of additives had a significant influence on the thermal conductivity as compared to the control pure PMMA sample. $\mathrm{SnO}_{2} \mathrm{NPs}$ function as an enhancement nano-additive to increase composite ability of heat transfer and increase thermal conductivity of the prepared composite, due to their better conductivity properties. As shown in Figure-8, the thermal conductivity values were increased from $0.216 \mathrm{~W} / \mathrm{m} . \mathrm{k}$ for the control case to $0.332 \mathrm{~W} / \mathrm{m} . \mathrm{k}$ for $\mathrm{PMMA} / 1 \% \mathrm{SnO}_{2}$ and $0.372 \mathrm{~W} / \mathrm{m} . \mathrm{k}$ for $\left(\mathrm{PMMA} / 2 \% \mathrm{SnO}_{2}\right)$, indicating an $\mathrm{SiO}_{2} \mathrm{NPs}$ percentage-dependent increase.

A better conductivity value was also observed for the hybrid reinforcement at $0.5 / 0.5$ of both additives, where the conductivity was increased to $0.229 \mathrm{~W} / \mathrm{m}$.k. Furthermore, the presence of the two additive probably worked to fill all the inside space and voids, leading to support heat transfer [25, 26]. On the other hands, at $2 \%$ additive for both $\mathrm{E}$ and $\mathrm{F}$ samples, a slight reduction in this property was observed. A similar situation was observed with ESP at the different percentages used. The latter behavior could be due to the poor interfacial adhesion within the polymer matrix upon the increase of the additive percentage, which agrees with previously reported observations [27]. This explains the notion that thermal property was increased in the presence of the metal fillers but was not proportionally increased as the filler concentration increased.

Moreover, for all percentages of hybrid additives used, the thermal conductivity values varied between rise and reduction, as illustrated in Fig.9. The maximum increase in this property was $53.34 \%$ and $71.29 \%$ for $1 \mathrm{wt} \%$ and $2 \mathrm{wt} \%$ of $\mathrm{SnO}_{2}$ NPs reinforcement respectively. While, a linear reduction in the thermal conductivity was clearly observed with the increase in the ESP additive percentage, which could be attributed to the weak interfacial bonding of both matrix-additives as the wt\% of ESP was increased [28]. 


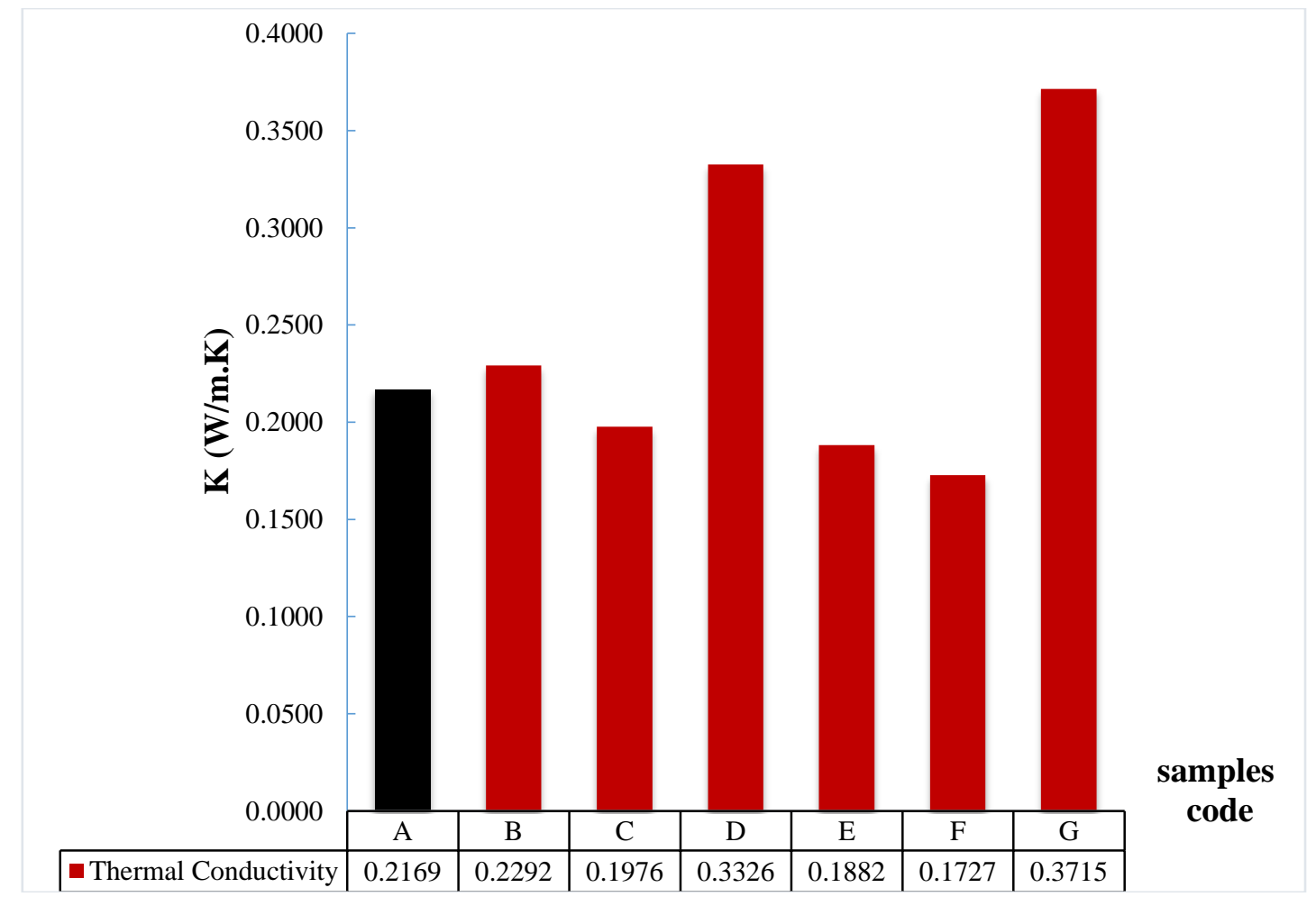

Figure 8-Comparison of thermal conductivity values as a function of different additives in the prepared samples.

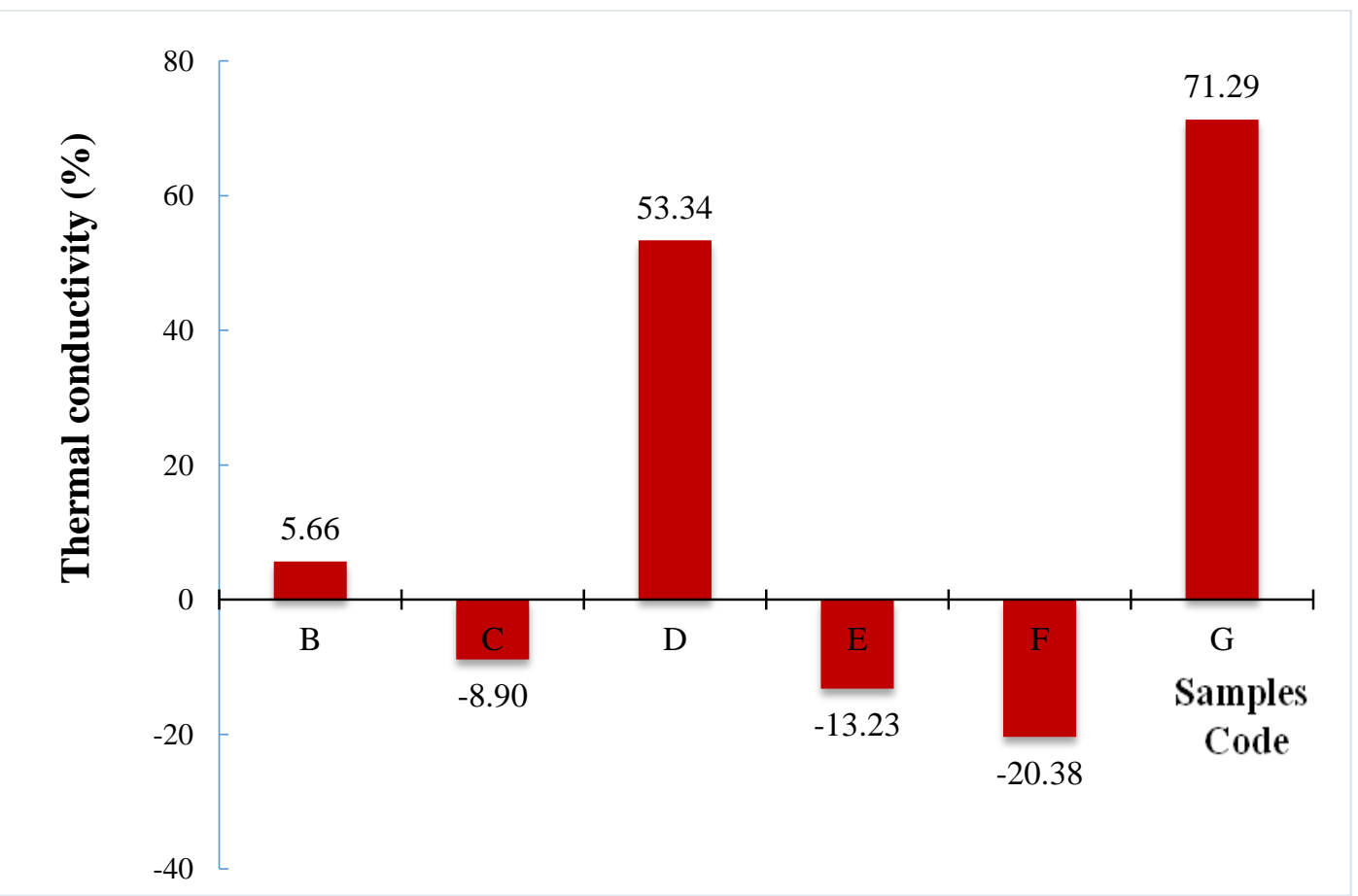

Figure 9-Thermal conductivity magnitude variation as a function of different additives in the prepared samples.

\subsection{Vickers hardness evaluation}

The results of this test showed a significant variation in the mean micro-hardness values among all the prepared samples (control PMMA, pure composites, and hybrid composite). The control PMMA samples exhibited a mean surface hardness of 8.25. Comparing this value with those of all the reinforced samples, an obvious trend of surface hardness increase was recorded with the increase in 
the additive weight percentage. Figure-10 illustrates the details of these results, where a noticeable increase in the surface hardness was recorded with the increase of ESP percentage (11.76 and 19.56 for (1\% and 2\%, respectively). While lower increase in surface hardness was observed using $\mathrm{SnO}_{2}$ NPs (12.35 and 13.30 for $1 \%$ and $2 \%$, respectively). For the hybrid composite, a relatively similar increase for both $1 \%$ and $2 \%$ (11.31 and 12.08 , respectively) was found. The observed increase in the surface hardness could be explained by the possibility that, during composite fabrication, a considerable amount of these additives resulted in stiffing the matrix polymer. The used additives possess better elastic modulus value than that of the matrix (control PMMA). Hence, much more resistance was provided by these additives to prevent the indenter from penetrating the sample surface at that specific load, compared with that of the control PMMA sample [29, 30].

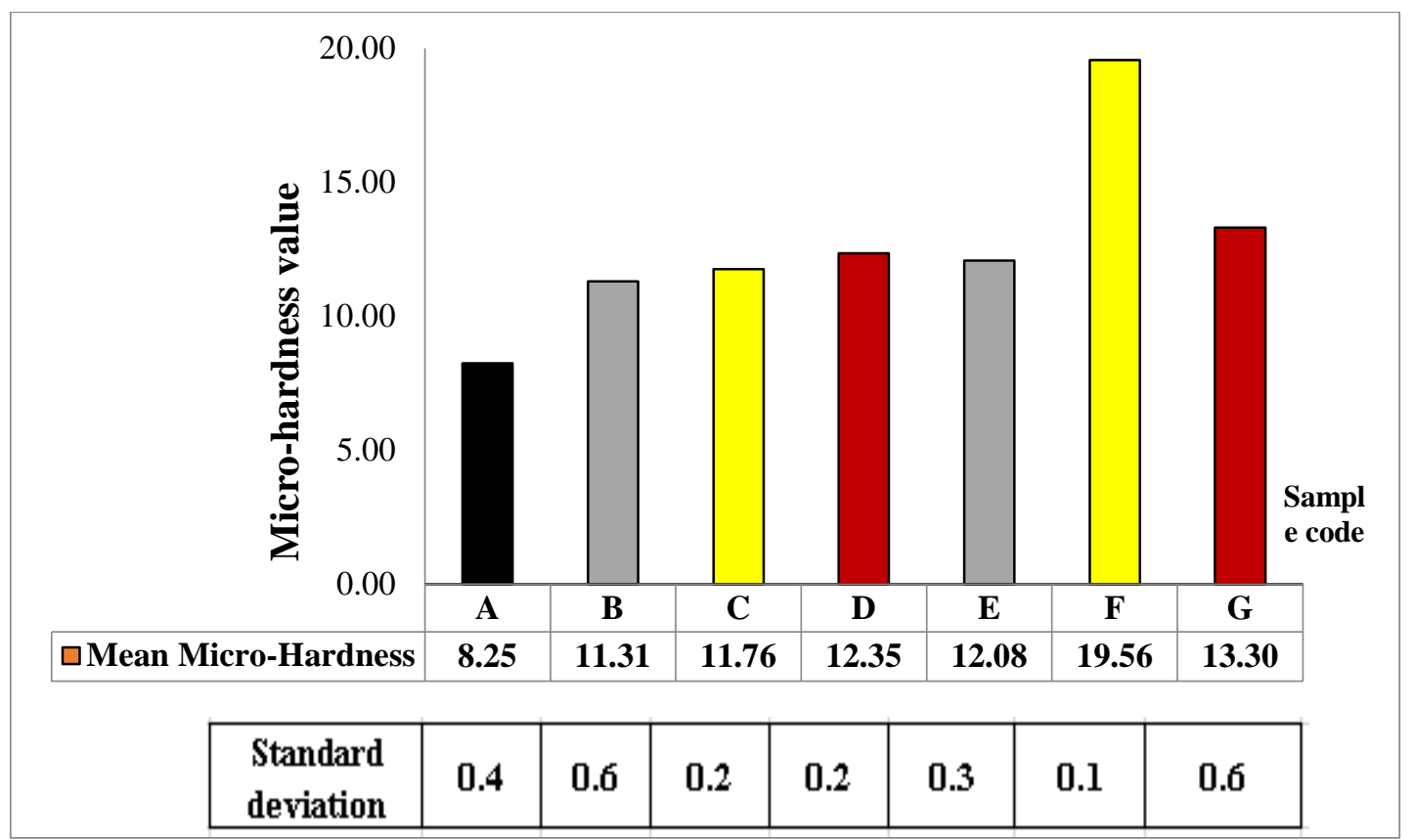

Figure 10-Descriptive statistics of the surface hardness test as a function of different additives in the prepared samples.

\subsection{Water absorption evaluation}

Water absorption behavior of all composites as function of time was also investigated. Figure-11 shows the variation of water absorption ratio versus exposure time for all prepared samples at different additive weight percentages and in both pure and hybrid composites. It is clear that both $\mathrm{G}$ and $\mathrm{F}$ samples, which contain the higher percentages of ESP and $\mathrm{SnO}_{2}$, respectively, showed higher water absorption values. Also, these two samples possessed better D coefficients, as illustrated in Table- 2 . This could be explained by the notion that the higher the filler content in the prepared composite the more absorbed water is observed. The agglomeration in the composite is increased as the filler content is increased, due to some difficulties in achieving homogeneous dispersion at high percentage of addition [31]. Both pure and hybrid composites showed better behavior compared to the control PMMA. Also, for both types of composites, the higher the additive percentage the higher D value was achieved. $F$ and $G$ samples showed D values of 21.43 and $21.71 \mathrm{~m}^{2} / \mathrm{sec}$, respectively, while the control PMMA resulted in a value of only $11.53 \mathrm{~m}^{2} / \mathrm{sec}$. Finally, after performed this test it must recorded that all the samples with all condition (pure and with additives) did not show any signs of swelling, dimension change, or even color change during water exposure time. 


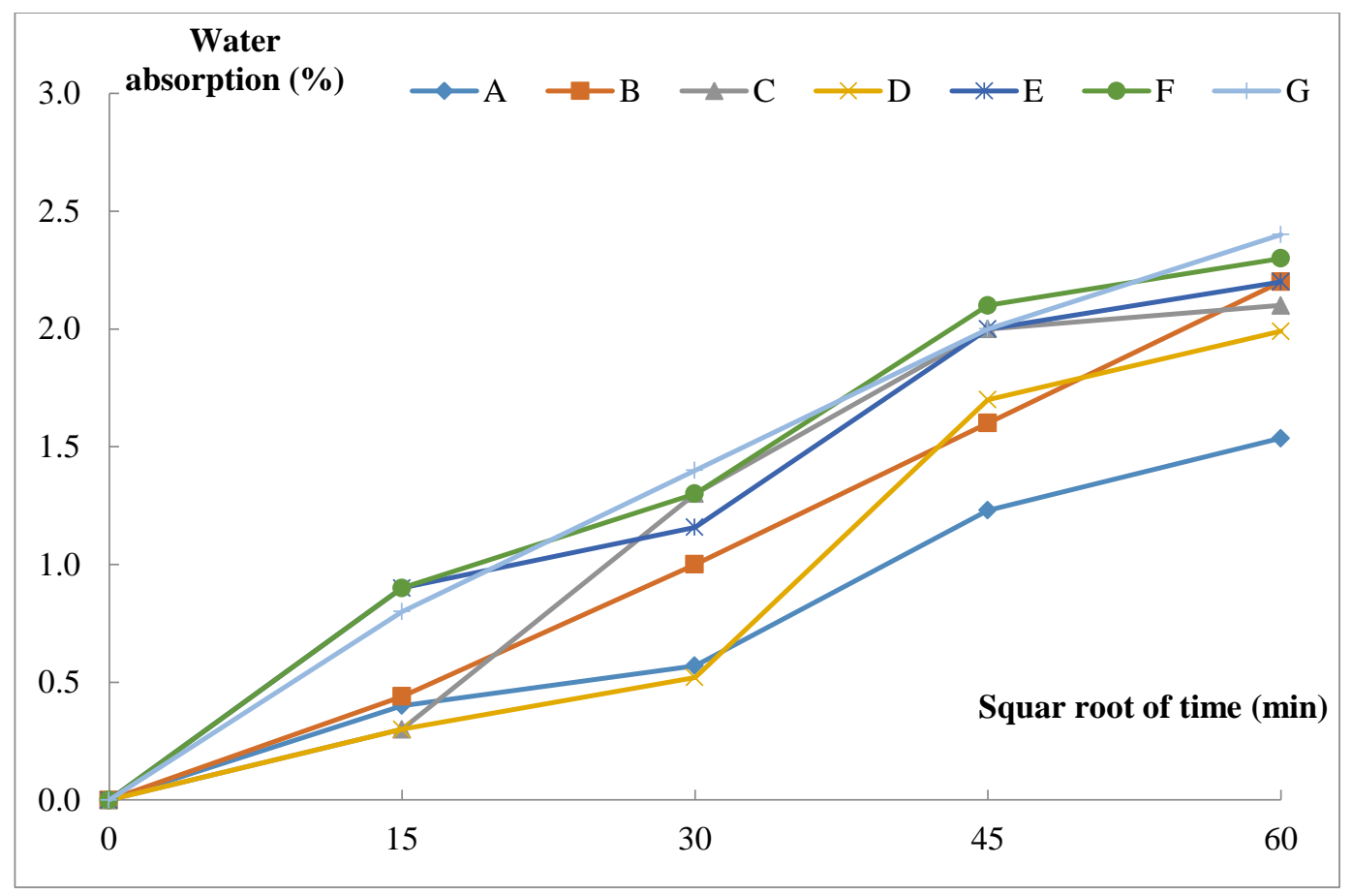

Figure 11-Effects of exposure time on water absorption of PMMA/ ESP and $\mathrm{PMMA} / \mathrm{SnO}_{2}$ pure and hybrid composites at different weight percentages.

Table 2-Measured values of D for the prepared samples

\begin{tabular}{|c|c|c|}
\hline Sample code & $\mathbf{S}$ & $\mathbf{D}(\mathbf{m} \mathbf{2} / \mathbf{s e c})$ \\
\hline A & 1.5 & 11.532 \\
\hline B & 2.2 & 16.971 \\
\hline C & 2.1 & 18.454 \\
\hline D & 1.9 & 13.703 \\
\hline E & 2.2 & 19.906 \\
\hline F & 2.3 & 21.429 \\
\hline G & 2.4 & 21.707 \\
\hline
\end{tabular}

\section{Conclusions}

With the limitations of this research, several conclusions could be achieved. The thermal properties of conventional acrylic denture was enhanced significantly by the addition of libratory prepared $\mathrm{SnO}_{2}$ NPs, in a weight percentage-dependent manner. The maximum value of thermal conductivity was $71 \%$ for $2 \mathrm{wt} \% \mathrm{SnO}_{2}$. The highest energy absorption was found at $1 \mathrm{wt} \%$ of the prepared composite. Surface hardness was increased as the additive wt\% was increased using all types of additives. Good improvement was found in micro-hardness, especially using ESP/PMMA composite as compared to the control PMMA. The higher the additive weight percentage added to the prepared composite the higher the water absorption measured. Hence, diffusion coefficient value was increased in respect to the additive percentage. Finally, no signs of swelling, dimension change, or color change noticed in all samples during water exposure. 


\section{References}

1. X.Xu, L. He, B. Zhu, J. Li, 2017. Polym. Chem. 8(2017): 807-823.

2. Phoenix, R. D., Mansueto, M. A., Ackerman, N. A. and Jones, R. E. 2004. Evaluation of mechanical and thermal properties of commonly used denture base resins, Journal ofProsthodontics, 13(1): 17-27.

3. McCabe JF, Walls AWG. 2008. Applied dental material, $9^{\text {th }}$ edition. Black well Scientific publication, London 2008;110-23.

4. Messersmith Phillip B, Ales O. and Sara L. 1998. New acrylic resin composite with improved thermal diffusivity. J Prosthet Dent; 79(3): 278-84.

5. Kapur KK, Fisher EE. 1981. Effect of denture base thermal conductivity on gustatory response. $J$ Prosthet Dent.1981; 46: 603-9

6. Navidfar, A., Azdast, T. and Karimzad, G. 2016. A. Influence of processing condition and carbon nano tube on mechanical properties of injection molded multi-walled carbon nanotube/poly (methyl methacrylate) nanocomposites. J. Appl. Polym. Sci.,133. http://onlinelibrary.wiley.com/doi/10.1002/app.43738/full.

7. Esmaili, P., Azdast, T., Doniavi, A., Hasanzadeh, R., Mamaghani, S. and Eungkee Lee, R. 2015. Experimental investigation of mechanical properties of injected polymeric nanocomposites containing multi-walled carbon nanotubes according to design of experiments. J.Sci.Technol. Compos, 2: 67-74.

8. Hasanzadeh, R., Azdast, T., Doniavi, A. and Esmaili, P. 2015. Experimental study on mechanical properties of polyamide-6/multi-walled carbon nanotubes nanocomposite foams. Int. J. Biol. Pharm. Allied Sci., 4: 208-223

9. Safarabadi, M. and Khansari, N., Rezaei, 2014. An experimental investigation of HA/Al2O nanoparticles on mechanical properties of restoration materials. Eng. Solid Mech., 2: 173-182.

10. Shirkavand, S. and Moslehifard, E. 2014. Effect of TiO nanoparticles on tensile strength of dental acrylic resins. J. Dent. Res. Dent. Clin. Dent. Prospects, 8: 197.

11. Sodagar, A., Bahador, A., Khalil, S., Shahroudi, A.S. and Kassaee, M.Z. 2013. The effect of TiO2 and $\mathrm{SiO}_{2}$ nanoparticles on flexural strength of poly (methyl methacrylate) acrylic resins. $J$. Prosthodont. Res., 57: 15-19.

12. Yerro, O., Radojević, V., Radović, I., Kojović, A., Uskoković, P.S., Stojanović, D.B. and Aleksić, R., 2016. Enhanced thermo-mechanical properties of acrylic resin reinforced with silanized alumina whiskers. Ceramics International, 42(9), pp.10779-10786.

13. Abdulhamed, A.N. and Mohammed, A.M. 2010. Evaluation of thermal conductivity of alumina reinforced heat cure acrylic resin and some other properties. J Bagh Coll Dent, 22(3): 1-6.

14. Zaynab N. Rasheed and Samah M. Hussain, 2019. Impact strength behaviour of PMMA denture base through addition of different nanoparticles after immersion in some nutrition liquids, Iraqi Journal of Physics, 17(41): 40-50.

15. Aljafery, A.M.A. 2018. Flexural Resistance and Impact Resistance of High-Impact Acrylic Resin with Addition of TiO2-Al2O3 Nanoparticles. Nano Biomed. Eng, 10(1): 40-45.

16. SihamaIssa Salih1, JawadKadhim Oleiwil and ArkanSaad Mohamed, 2018. "Investigation of mechanical properties of PMMA composite reinforced with different types of natural powders "ARPN Journal of Engineering and Applied Sciences, 13(22), NOVEMBER.

17. Samah M. Hussein, Zaynab N. Rasheed, 2019. "Nano-reinforcement effect on PMMA denture base mechanical properties after immersing in different solution", Iraqi journal of science, Special issue:57-68, DOI: 10.24996/ijs.2019.S.I.10

18. Madzlan Aziz, Saad Saber Abbas, Wan Rosemaria Wan Baharom. 2013. Size-controlled synthesis of $\mathrm{SnO}_{2}$ nanoparticles by sol-gel method. Materials Letters, 91: 31-34.

19. Rashed T. Rasheed,andSariya D. Al-Algawi. 2016. Annealing Effect of $\mathrm{SnO}_{2}$ Nanoparticles Prepared by the Sol-Gel Method. Journal of Advanced Physics. 5: 236-240.

20. Aziz,M., Saber Abass, s.,and Wan Baharom, W.R. 2013. "Size-controlled synthesis of $\mathrm{SnO}_{2}$ nanoparticles by sol-gel method. Materials letters, 91: 31-34.

21. Tolf G. and Clarin P. 1981. "Fiber Composite Hybrid Materials", Applied Science Publisher LTD, London. 
22. Pfeiffer, P. and Rosenbauer, E. U. 2004. Residual methyl methacrylate monomer, water sorption, and water solubility of hypoallergenic denture base materials. The Journal of prosthetic dentistry, 92(1): 72-78.

23. Abirami Dhanabalan, Xifei Li, RichaAgrawal, Chunhui Chen and Chunlei Wang. 2013. Fabrication and Characterization of $\mathrm{SnO}_{2} /$ Graphene Composites as High Capacity Anodes for LiIon Batteries. Nanomaterials, 3: 606-614.

24. N. Nadaud, N. Lequeux, M. Nanot, J. Jové, and T. Roisnel. 1998. Structural Studies of Tin-Doped Indium Oxide (ITO) and $\mathrm{In}_{4} \mathrm{Sn}_{3} \mathrm{O}_{12}$. Journal of Solid State Chemistry. 135(1): 140-148.

25. Sandler, S.R., Karo, W., Bonesteel, J. and Pearce, E.M. 1998. Polymer synthesis and characterization: A laboratory manual. Elsevier.

26. Belhaneche-Bensemra N., Bedda A. 2001. "Analysis of Structure Properties Relationship of PVCPMMA Blends", Ann. Chim., 26(3): 79-90.

27. Schajpal S. B. and Sood V. K. 1989."Effect of Metal Fillers on Some Physical Properties of Acrylic Resins", Jour. Pros. Dent.; 61: 746-751.

28. Hassan, S.B., Aigbodion, V.S. and Patrick, S.N. 2012. Development of polyester/eggshell particulate composites. Tribology in industry, 34(4): 217-225.

29. Samrand Rashahmadi, Rezgar Hasanzadeh \& Sina Mosalman 2017. Improving the Mechanical Properties of Poly Methyl Methacrylate Nanocomposites for Dentistry Applications Reinforced with Different Nanoparticles, Polymer-Plastics Technology and Engineering, DOI: 10.1080/03602559.2017.1289402.

30. Anjali, Razia Malik, Shivani Bhandari, Ankur Pant, Arpit Saxena, Seema, Nitin Kumar, Nikhil Chotrani, Dheeraj Gunwant*, P. L. Sah. 2017. "Fabrication and Mechanical Testing of Egg Shell Particles Reinforced Al-Si Composites", International Journal of Mathematical, Engineering and Management Sciences, 2(1): 53-62, 2017

31. Salih, S.I., Oleiwi, J.K. and Ali, H.M. 2019. Development the Physical Properties of Polymeric Blend (SR/PMMA) by Adding various Types of Nanoparticles, Used for Maxillofacial Prosthesis Applications. Engineering and Technology Journal, 37(4 A): 120-127. 